\title{
Feasibility of identifying important changes in care management resulting from cardiovascular magnetic resonance (CMR) using hospital episode data in patients who activate the primary percutaneous coronary intervention (PPCl) pathway
}

Maria Pufulete ${ }^{1 *}$ D, Jessica Harris ${ }^{1}$, Stephen Dorman², Lynn Cook³ ${ }^{3}$ Chiara Bucciarelli-Ducci' ${ }^{2}$ John P. Greenwood ${ }^{4}$, Richard Anderson ${ }^{5}$, Rachel Brierley ${ }^{1}$ and Barnaby C. Reeves ${ }^{1}$

\begin{abstract}
Background: We determined whether it is feasible to identify important changes in care management resulting from cardiovascular magnetic resonance (CMR) in patients who activate the primary percutaneous coronary intervention (PPCI) pathway from hospital episode data, in order to construct a composite primary outcome (hypothesised to reduce the risk of major adverse cardiac-related events, MACE) to compare patients exposed to CMR or not.

Methods: We used Hospital Episode Statistics (HES) and Patient Episode Database for Wales (PEDW) to identify clinical events that reflected important changes in management in the year following the index admission in five subgroups of patients who activated the PPCI pathway recruited as part of a feasibility cohort study $n=1655$ with HES/PEDW data). For all subgroups, we identified frequency of events and time to the first event for each change in management.

Results: We identified all clinical events (new diagnoses, additional diagnostic tests and procedures) except for medication prescriptions. Diagnostic tests were underestimated because most are carried out in outpatient clinics and outpatient datasets had missing procedure codes for $74 \%$ of patients (some tests done in hospital may also not be recorded). We successfully tabulated frequencies of events and distributions of times to first event for most changes in management by CMR status and in CMR / non CMR centres.

Conclusions: It is feasible to identify changes in care management between patients who have / do not have CMR within relevant patient subgroups. Further work to derive a weighting algorithm is required before attempting to combine the events in a composite endpoint.
\end{abstract}

Keywords: Cardiovascular magnetic resonance (CMR), Primary percutaneous coronary intervention (PPCI), Hospital Episode Statistics (HES), England, Patient episode database Wales (PEDW)

\footnotetext{
* Correspondence: maria.pufulete@bristol.ac.uk

${ }^{1}$ Clinical Trials and Evaluation Unit, University of Bristol, Level 7, Bristol Royal

Infirmary, Queen's Building, Bristol, UK

Full list of author information is available at the end of the article
} 


\section{Background}

Prospective patient registries provide a real-world view of clinical practice and patient outcomes including safety (http://www-new.njrcentre.org.uk/njrcentre/Home/ tabid/36/Default.aspx) [1-4]. They can also be useful to estimate comparative effectiveness providing that they can accrue sufficient data quickly enough to estimate treatment effects with adequate precision. In patients who have attended an Emergency Department (ED) with ST-elevation myocardial infarction (STEMI), the frequency of subsequent major adverse cardiac-related events (MACE) has decreased markedly over the past 20 years [5-8]. This decrease has been achieved mainly through improvements in management and use of evidence-based pharmacological and interventional therapies. Therefore, studies evaluating the effectiveness of alternative management pathways in STEMI populations using MACE as the primary outcome require large sample sizes to achieve satisfactory power.

We conducted a study to determine the feasibility of setting up a registry linking data collected during usual care to assess the clinical and cost-effectiveness of cardiovascular magnetic resonance (CMR) in patients who activate the primary percutaneous coronary intervention (PPCI) pathway [9]. Although a longterm objective of the registry would be to compare the incidence of MACE in patients who do or do not have CMR after the index event, the relatively low frequency of MACE after PPCI in this population (about $13 \%$ [9]) means that the study would have to accrue over 27,000 subjects to detect a clinically important (assumed to be 10\%) relative reduction in the incidence of MACE with $90 \%$ power. Therefore, a key objective of the feasibility study was to define a primary composite outcome, acceptable to cardiologists and other stakeholders (e.g. healthcare providers) as representing a clinically important change in management (e.g. expected to prevent future MACE and change practice/commissioning) resulting from CMR that could be used for the registry in the medium term.

We identified important changes in management resulting from CMR (and the specific patient subgroups these changes in management relate to) using formal consensus methods [10]. In this paper, we report on whether it is feasible to identify these changes in management during follow up from routinely collected hospital episode data (Hospital Episode Statistics, HES; Patient Episode Database Wales, PEDW). HES is commonly used for outcome ascertainment in cardiovascular disease populations and has been shown to be valid and reliable in both registries and clinical trials [11-14]. If successful, this process of ascertainment could be used to define a primary composite outcome to compare groups of PPCI patients exposed to CMR or not.

\section{Methods}

\section{Patient recruitment for the feasibility study}

Patients were recruited from four NHS hospitals in England and Wales with 24/7 PPCI services (Bristol, Leeds, Swansea and Cardiff) between May 2013 and September 2014. Two hospitals (Bristol and Leeds) were defined as "CMR centres", i.e. hospitals that had a local dedicated CMR service; the other two (Swansea and Cardiff) were defined as "non-CMR centres", i.e. hospitals without access to a local dedicated CMR service. Patient identification, recruitment and consent have been described previously [9]. Patients entered the cohort at the point they activated the PPCI pathway (i.e. had an emergency angiogram), regardless of whether PPCI was carried out or not.

\section{Formal consensus to identify important changes in management (and the subgroups of patients to which these referred)}

We used formal consensus (literature review and cardiologist expert opinion) to formulate consensus statements about important changes in management arising from the use of CMR in patients who activate the PPCI pathway. The formal consensus process has been described elsewhere [10]. Five patient subgroups were identified as potentially benefitting from CMR, namely patients who: i) have an out of hospital cardiac arrest (OHCA); ii) have "normal" (unobstructed) coronary arteries; iii) develop LV thrombus after STEMI; iv) have multivessel disease (MVD, defined as 2 or more vessels with $>50 \%$ stenosis pre-PCI); and v) underwent PPCI and have a suspected poor prognosis (assumed to be identifiable from CMR imaging biomarkers).

\section{Identifying the patient subgroups defined through formal consensus}

Three of the patient subgroups (patients who underwent PPCI, patients with unobstructed coronary arteries and patients with MVD) were defined using data collected at cohort entry (related to the index admission). Two subgroups, patients who had had an OHCA and those who went on to develop LV thrombus, could only be identified from HES / PEDW (data collected at cohort entry relating to the presence or absence of these conditions were missing for a large proportion of participants), using ICD-10 diagnosis codes I46 "Cardiac arrest" and I23.6 "Thrombosis of atrium, auricular appendage and ventricle as current complications following acute myocardial infarction", respectively.

\section{Identifying CMR exposure}

CMR exposure was defined as documentation of CMR in the imaging dataset (collected from "CMR centres") within 10 weeks of their index admission; this time 
frame was chosen to capture both urgent and nonurgent (out-patient) CMR scans.

\section{Identifying the key clinical "events" that reflected the important changes in management}

Initially, cardiologist members of the clinical team identified the key clinical "events" that they expected to reflect the important changes in management characterised through the consensus process. We then used HES and PEDW inpatient and outpatient data to ascertain these changes in management, compiling a list of diagnosis and procedure codes (International Classification of Disease, ICD-10; Office of Population Censuses and Surveys Classification of Interventions and Procedures, OPCS) representing the key clinical events with the help of a clinical coder. Most changes in management were defined by Boolean combinations of multiple ICD-10 / OPCS codes; for example, the "use of additional diagnostic tests during follow-up" was ascertained by OPCS codes for echocardiography (K58.5 OR U20.1 OR U20.2 OR U20.3 OR U20.4 OR U20.5), single photon emission computed tomography (SPECT) (U21.4); positron emission tomography (PET; U10.4 OR U21.3 OR U36.2), intra vascular ultrasound (IVUS; K51.2 OR L726), pressure-wire (K63.4 OR K63.5 OR K63.6) AND K51.8 AND Y44.2 AND Y53), radionuclide angiocardiography (U10.5) or computed tomography angiography (U10.2). We identified the key clinical events relating to the changes in management for each patient subgroup separately. The full code list used to identify changes in management is shown in the Additional file 1. For those patients who underwent CMR, any outpatient appointments that occurred between the index admission and the date of CMR were excluded. We did not use Accident and Emergency (A\&E) data because most of the changes in management identified in the formal consensus process were deemed unlikely to have occurred in the A\&E setting and diagnoses and procedures are not well coded in the A\&E dataset.

\section{Statistical analysis}

Data were analysed using Stata/IC (V13, StataCorp LP, Texas, USA). The analysis was descriptive, with quantitative results expressed as counts and percentages. We calculated the frequencies of all events representing changes in management (e.g. number of new diagnoses, additional diagnostic tests, number / rate of outpatient appointments, etc.) in patients who did / did not receive CMR for each patient subgroup in the 12 months following the index admission.

We also calculated the time to first event (in participants in whom an event occurred) for each change in management (e.g. time to first new diagnosis, time to the first diagnostic test, time to next revascularisation in patients with multivessel disease, etc.) and summarised these with medians and interquartile ranges (IQR). Dates of events were available for all events, which allowed us to describe the time to first event for each change in management and for all subgroups, and to visualise the data using Kaplan Meier curves and other time to event plots. We did not compare differences between patients who did / did not receive CMR in any of the subgroups because this was not an objective; we were primarily interested in the feasibility of identifying relevant events and the frequencies of events in most subgroups were small. We did not attempt to derive a composite outcome because further research would be required to derive an appropriate weighting algorithm to combine the different clinical events.

\section{Results}

We recruited 1670 patients across the four hospitals, of whom 1655 (99\%) had HES/PEDW data. Of these, 89\% of patients underwent PPCI; 11\% were found to have unobstructed coronary arteries, $44 \%$ of patients had MVD, $7 \%$ had out-of-hospital cardiac arrest (OHCA) and $0.06 \%$ had left ventricular (LV) thrombus. The proportions of patients in each subgroup were similar between hospitals, except for patients with MVD (19\% in hospital D vs. 48,51 and $53 \%$ in hospitals $A, B$ and C, respectively) (Table 1).

\section{Identifying changes in management in hospital episode statistics data}

The ICD-10 diagnosis codes and OPCS procedure codes of the clinical events representing important changes in management resulting from CMR are shown in Table 2. We successfully identified the events described in the table, except for medications; these are not available in HES/PEDW data. We were also unable to obtain data about medications on discharge for a large proportion of the cohort. Therefore, we could not identify changes in medication prescribed during follow up. All relevant non-ischaemic diagnoses were identifiable in admitted patient care data, as were procedures such as implantation of devices, repeat revascularisation and additional diagnostic tests. In contrast, we could not identify any of these from outpatient care data because ICD-10 diagnosis codes and OPCS procedure codes associated with each outpatient episode were missing for a large proportion of patients. All outpatient visits should have a diagnostic code associated with the visit. However, for at least one of their visits, $25 \%$ of patients had missing ICD-10 diagnosis codes, $74 \%$ of patients had missing OPCS procedure codes (which may indicate either that no procedure took place or that a procedure took place but was not recorded), and $74 \%$ of patients had unknown "Unknown and unspecified causes of morbidity" as their ICD-10 diagnosis code). 
Table 1 Frequency of patient subgroups by hospital

\begin{tabular}{llllll}
\hline & $\begin{array}{l}\text { Hospital A } \\
(n=758)\end{array}$ & $\begin{array}{l}\text { Hospital B } \\
(n=272)\end{array}$ & $\begin{array}{l}\text { Hospital C } \\
(n=316)\end{array}$ & $\begin{array}{l}\text { Hospital D } \\
(n=309)\end{array}$ & $\begin{array}{l}\text { Total } \\
(n=1655)\end{array}$ \\
\hline PPCl & $651(86 \%)$ & $246(90 \%)$ & $291(92 \%)$ & $279(90 \%)$ & $1467(89 \%)$ \\
MVD & $361(48 \%)$ & $138(51 \%)$ & $169(53 \%)$ & $58(19 \%)$ & $726(44 \%)$ \\
LV thrombus after PPCl & $1(0.13 \%)$ & 0 & 0 & 0 & $1(0.06 \%)$ \\
Unobstructed coronary arteries & $107(14 \%)$ & $26(10 \%)$ & $25(8 \%)$ & $30(10 \%)$ & $18(11 \%)$ \\
OHCA & $57(8 \%)$ & $22(8 \%)$ & $16(5 \%)$ & $14 \%)$ & $109(7 \%)$ \\
\hline
\end{tabular}

Percentages do not add up to 100 because some patients were in more than one subgroup

$L V$ left ventricular, MVD multivessel disease, $O H C A$ out of hospital cardiac arrest, $P P C I$ primary percutaneous coronary intervention

Important changes in management by CMR status and in CMR / non CMR centres

Tables 3 and 4 show the number of events and time to first event representing changes in management in the relevant patient subgroups identified in HES / PEDW up to 12 months after the index admission by CMR status and in CMR vs non CMR centres, respectively. It was possible to observe differences in both the frequency of events and time to first event by CMR status or in CMR vs non CMR centres. For example, across all subgroups, patients in CMR centres had more outpatient appointments and an earlier time to first appointment (see Fig. 1

Table 2 Data sources and definitions used for identifying changes in management up to 12 months after the index admission

\begin{tabular}{|c|c|c|c|}
\hline $\begin{array}{l}\text { Important change in } \\
\text { management resulting } \\
\text { from } \mathrm{CMR}^{\mathrm{a}}\end{array}$ & $\begin{array}{l}\text { Patient } \\
\text { subgroup }\end{array}$ & Data source & $\begin{array}{l}\text { ICD-10 diagnosis codes and OPCS procedure codes (up to } 12 \text { months } \\
\text { after the index admission) }\end{array}$ \\
\hline $\begin{array}{l}\text { New diagnosis (non- } \\
\text { ischaemic) }\end{array}$ & $\begin{array}{l}\text { Unobstructed } \\
\text { coronary } \\
\text { arteries } \\
\text { OHCA }\end{array}$ & $\begin{array}{l}\text { HES / PEDW admitted } \\
\text { patient care data }\end{array}$ & $\begin{array}{l}\text { Any record of the following: } \\
\text { - Takotsubo cardiomyopathy (I42.8 AND F43.8) } \\
\text { - Myocarditis (I51.4) } \\
\text { - Pericarditis (I30 OR I31.0 OR I31.1 OR I31.9 OR I32.0 OR I32.1 OR } \\
\text { I32.8 OR I01.0 OR I02.0 OR I09.2) } \\
\text { - Endocarditis (I33.9) } \\
\text { - Coronary spasm (I20.1) }\end{array}$ \\
\hline Changes in medication & $\begin{array}{l}\text { PPCI } \\
\text { MVD } \\
\text { LV thrombus after PPCI } \\
\text { Unobstructed coronary } \\
\text { arteries } \\
\text { OHCA }\end{array}$ & Not available & Not available \\
\hline $\begin{array}{l}\text { Additional } \\
\text { diagnostic tests }\end{array}$ & $\begin{array}{l}\mathrm{PPCl} \\
\text { MVD } \\
\text { LV thrombus after PPCI } \\
\text { Unobstructed coronary } \\
\text { arteries } \\
\text { OHCA }\end{array}$ & $\begin{array}{l}\text { HES / PEDW admitted } \\
\text { patient care data and } \\
\text { outpatient care data }\end{array}$ & $\begin{array}{l}\text { Any record of the following: } \\
\text { - Echocardiography (K58.5 OR U20.1 OR U20.2 OR U20.3 OR U20.4 OR } \\
\text { U20.5) } \\
\text { - Single photon emission computed tomography (SPECT) (U21.4) } \\
\text { - Positron emission tomography (PET) scans (U10.4 OR U21.3 OR U36.2) } \\
\text { - Intravascular ultrasound (IVUS) (K51.2 OR L726) } \\
\text { - Pressure wire ((K63.4 OR K63.5 OR K63.6) AND K51.8 AND Y44.2 AND } \\
\text { Y53) } \\
\text { - Radionuclide angiocardiography (U10.5) } \\
\text { - Computed tomography angiography (U10.2) }\end{array}$ \\
\hline $\begin{array}{l}\text { Implantation } \\
\text { of devices }\end{array}$ & $\begin{array}{l}\mathrm{PPCl} \\
\mathrm{OHCA}\end{array}$ & $\begin{array}{l}\text { HES / PEDW admitted } \\
\text { patient care data }\end{array}$ & $\begin{array}{l}\text { Any record of the following: } \\
\text { - Cardiac resynchronization therapy (CRT) (K60.7 OR K61.7 OR K59.6) } \\
\text { - Implantable cardioverter defibrillator (ICD) (K59 OR K72) }\end{array}$ \\
\hline $\begin{array}{l}\text { Revascularisation } \\
\text { (PCl or CABG) } \\
\text { within } 3 \text { months }\end{array}$ & MVD & $\begin{array}{l}\text { HES / PEDW admitted } \\
\text { patient care data }\end{array}$ & $\begin{array}{l}\text { Any record of the following (up to } 3 \text { months after the index admission): } \\
\text { - PCI (K49 OR K50 OR K75) } \\
\text { - CABG (K40 OR K41 OR K42 OR K43 OR K44 OR K45 OR K46) }\end{array}$ \\
\hline $\begin{array}{l}\text { Frequency of } \\
\text { cardiology } \\
\text { outpatient } \\
\text { appointments }\end{array}$ & $\begin{array}{l}\text { PPCI } \\
\text { MVD } \\
\text { LV thrombus after PPCI } \\
\text { Unobstructed coronary } \\
\text { arteries } \\
\text { OHCA }\end{array}$ & $\begin{array}{l}\text { HES / PEDW } \\
\text { outpatient care data }\end{array}$ & $\begin{array}{l}\text { Rate of outpatient visit attended (count of visits divided by follow-up time) } \\
\text { where the treatment speciality in which the consultant responsible was } \\
\text { working during the period of care was Cardiology (code }=320 \text { ). }\end{array}$ \\
\hline
\end{tabular}

CMR cardiovascular magnetic resonance, HES Hospital Episode Statistics, ICD-10 International Classification of Diseases, $L V$ left ventricular, OHCA out of hospital cardiac arrest, MVD multivessel disease, OPCS Office of Population Census and Surveys Classification of Intervention and Procedures, PEDW Patient Episode Database Wales, $\mathrm{PPCl}$ primary percutaneous coronary intervention

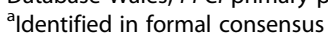


Table 3 Events and time to first event representing changes in management identified in HES / PEDW inpatient and outpatient data up to 12 months after the index admission by CMR status

\begin{tabular}{|c|c|c|c|c|c|c|c|c|c|c|c|}
\hline \multirow{3}{*}{$\begin{array}{l}\text { Patient } \\
\text { subgroup }\end{array}$} & \multicolumn{11}{|c|}{$\begin{array}{l}\text { Number of events (\%) identified in HES / PEDW up to } 12 \text { months after the index admission and number of days (median and IQR) until } \\
\text { the first event after the index admission }\end{array}$} \\
\hline & \multicolumn{2}{|c|}{$\begin{array}{l}\text { New diagnoses } \\
\text { (e.g. Takotsubo, } \\
\text { myocarditis, } \\
\text { pericarditis, } \\
\text { endocarditis, } \\
\text { coronary spasm) }\end{array}$} & \multirow[t]{2}{*}{$\begin{array}{l}\text { Changes in } \\
\text { medication }\end{array}$} & \multicolumn{2}{|c|}{$\begin{array}{l}\text { Additional diagnostic } \\
\text { tests (ECHO, SPECT, } \\
\text { PET, IVUS, pressure-wire, } \\
\text { radionuclide angiocardiography, } \\
C T \text { angiography) }\end{array}$} & \multicolumn{2}{|c|}{$\begin{array}{l}\text { Implantation of } \\
\text { devices (CRT or ICD) }\end{array}$} & \multicolumn{2}{|c|}{$\begin{array}{l}\text { Revascularisation } \\
(\mathrm{PCl} \text { or } \mathrm{CABG}) \\
\text { within } 3 \text { months }\end{array}$} & \multicolumn{2}{|c|}{$\begin{array}{l}\text { Cardiology } \\
\text { outpatient } \\
\text { appointments } \\
\text { (median, IQR) }\end{array}$} \\
\hline & $\begin{array}{l}\text { No. of } \\
\text { events }\end{array}$ & $\begin{array}{l}\text { No. of } \\
\text { days }\end{array}$ & & No. of events & No. of days & $\begin{array}{l}\text { No. of } \\
\text { events }\end{array}$ & $\begin{array}{l}\text { No. of } \\
\text { days }\end{array}$ & $\begin{array}{l}\text { No. of } \\
\text { events }\end{array}$ & $\begin{array}{l}\text { No. of } \\
\text { days }\end{array}$ & $\begin{array}{l}\text { No. of } \\
\text { events }\end{array}$ & $\begin{array}{l}\text { No. of } \\
\text { days }\end{array}$ \\
\hline$\overline{\mathrm{PPCl}}$ & & & No data & & & & & N/A & & & \\
\hline $\begin{array}{l}\text { CMR } \\
(n=152)\end{array}$ & 0 & - & & $16(11 \%)$ & $107(50,240)$ & 0 & - & & & $2(1,3)$ & $\begin{array}{l}64 \\
(34,99)\end{array}$ \\
\hline $\begin{array}{l}\text { No CMR } \\
(n=1312)\end{array}$ & $\begin{array}{l}10 \\
(1 \%)\end{array}$ & $16(8,20)$ & & $129(10 \%)$ & $74(27,171)$ & $6(0.5 \%)$ & $\begin{array}{l}147 \\
(48,171)\end{array}$ & & & $1(1,2)$ & $\begin{array}{l}55 \\
(33,95)\end{array}$ \\
\hline \multicolumn{12}{|l|}{ MVD } \\
\hline $\begin{array}{l}\text { CMR } \\
(n=104)\end{array}$ & 0 & - & & $11(11 \%)$ & $85(50,221)$ & 0 & - & $11(11 \%)$ & $\begin{array}{l}99 \\
(50,180)\end{array}$ & $2(1,3)$ & $\begin{array}{l}76 \\
(52,116)\end{array}$ \\
\hline $\begin{array}{l}\text { No CMR } \\
(n=622)\end{array}$ & $4(1 \%)$ & $13(8,110)$ & & $61(10 \%)$ & $62(24,130)$ & $4(1 \%)$ & $\begin{array}{l}147 \\
(84,169)\end{array}$ & $64(10 \%)$ & $\begin{array}{l}66 \\
(39,122)\end{array}$ & $1(1,2)$ & $\begin{array}{l}57 \\
(33,95)\end{array}$ \\
\hline \multicolumn{8}{|c|}{ LV thrombus after PPCI } & N/A & & & \\
\hline $\begin{array}{l}\text { CMR }(n= \\
\text { 1) }\end{array}$ & 0 & - & & 0 & - & 0 & - & & & $3(3,3)$ & $\begin{array}{l}66 \\
(66,66)\end{array}$ \\
\hline $\begin{array}{l}\text { No CMR } \\
(n=0)\end{array}$ & 0 & - & & 0 & - & 0 & - & & & - & - \\
\hline \multicolumn{12}{|c|}{ Unobstructed coronary arteries } \\
\hline $\begin{array}{l}\text { CMR }(n= \\
35)\end{array}$ & $1(3 \%)$ & $\begin{array}{l}358(358, \\
358)\end{array}$ & & $6(17 \%)$ & $93(58,140)$ & $1(3 \%)$ & $\begin{array}{l}78 \\
(78,78)\end{array}$ & & & $2(1,3)$ & $\begin{array}{l}67 \\
(52,121)\end{array}$ \\
\hline $\begin{array}{l}\text { No CMR } \\
(n=139)\end{array}$ & $1(1 \%)$ & $\begin{array}{l}180(180 \\
180)\end{array}$ & & $12(9 \%)$ & $123(78,284)$ & $3(2 \%)$ & $\begin{array}{l}245 \\
(71,262)\end{array}$ & & & $1(0,2)$ & $\begin{array}{l}67 \\
(44,106)\end{array}$ \\
\hline \multicolumn{12}{|l|}{$\mathrm{OHCA}$} \\
\hline $\begin{array}{l}\text { CMR }(n= \\
14)\end{array}$ & 0 & - & & $2(14 \%)$ & $110(79,141)$ & 0 & - & & & $1(1,3)$ & $\begin{array}{l}65 \\
(61,104)\end{array}$ \\
\hline $\begin{array}{l}\text { No CMR } \\
(n=95)\end{array}$ & $1(1 \%)$ & $48(48,48)$ & & 10 (11\%) & $62(48,93)$ & $5(5 \%)$ & $\begin{array}{l}126 \\
(71,167)\end{array}$ & & & $1(1,2)$ & $\begin{array}{l}64 \\
(41,99)\end{array}$ \\
\hline
\end{tabular}

CABG coronary artery bypass grafting, CMR cardiovascular magnetic resonance imaging, CRT cardiac resynchronisation therapy, ECHO echocardiography, HES Hospital Episode Statistics, ICD implantable cardioverter defibrillator, IVUS intravascular ultrasound, IQR interquartile range, LV left ventricular, MVD multivessel disease, OHCA out of hospital cardiac arrest, PET positron emission tomography, $P C I$ percutaneous coronary intervention, PEDW Patient Episode Database Wales, $P P C I$ primary percutaneous coronary intervention

for an example in the OHCA patient subgroup), and more frequent diagnostic tests and implantation of devices than patients in non CMR centres. Across most patient subgroups, patients in CMR centres and patients who had CMR seemed to have had an admission for an additional diagnostic test later than patients in nonCMR centres or patients who did not have CMR, respectively (see Fig. 2 for an example of CMR vs no CMR in the MVD patient group).

\section{Discussion}

\section{Main findings}

We have shown that it is feasible to identify clinical events expected to prevent future MACE and describe their frequencies and timing in relation to index PPCI pathway activation using only routinely collected NHS hospital episode data. We were able to identify relevant patient subgroups specified previously by combining data about the index event, which was obtained directly from hospitals, with admitted patient care (APC) hospital episode data. For each patient subgroup, we listed the "key clinical events" (e.g. new diagnoses or procedures, additional diagnostic tests, changes in medication, outpatient appointments) that reflected a change in management, identified through discussions with relevant clinicians. By looking at care pathways, where available, we confirmed that we captured all aspects of patient management in these key clinical events. We then compiled a list of the relevant ICD-10 diagnosis 
Table 4 Events and time to first event representing changes in management identified in HES / PEDW inpatient and outpatient data up to 12 months after the index admission in CMR vs. non CMR centres (hospitals A and B vs. hospitals C and D, respectively)

\begin{tabular}{|c|c|c|c|c|c|c|c|c|c|c|c|}
\hline \multirow{3}{*}{ Patient subgroup } & \multicolumn{11}{|c|}{$\begin{array}{l}\text { Number of events (\%) identified in HES / PEDW up to } 12 \text { months after the index admission and number of days (median } \\
\text { and IQR) until first event after the index admission }\end{array}$} \\
\hline & \multicolumn{2}{|c|}{$\begin{array}{l}\text { New diagnoses } \\
\text { (e.g. Takotsubo, } \\
\text { myocarditis, } \\
\text { pericarditis, } \\
\text { endocarditis, } \\
\text { coronary spasm) }\end{array}$} & \multirow[t]{2}{*}{$\begin{array}{l}\text { Changes in } \\
\text { medication }\end{array}$} & \multicolumn{2}{|c|}{$\begin{array}{l}\text { Additional diagnostic } \\
\text { tests (ECHO, SPECT, } \\
\text { PET, IVUS, pressure-wire, } \\
\text { radionuclide } \\
\text { angiocardiography, } \\
\text { CT angiography) }\end{array}$} & \multicolumn{2}{|c|}{$\begin{array}{l}\text { Implantation of } \\
\text { devices (CRT or ICD) }\end{array}$} & \multicolumn{2}{|c|}{$\begin{array}{l}\text { Revascularisation } \\
\text { (PCl or CABG) } \\
\text { within } 3 \text { months }\end{array}$} & \multicolumn{2}{|c|}{$\begin{array}{l}\text { Cardiology } \\
\text { outpatient } \\
\text { appointments } \\
\text { (median, IQR) }\end{array}$} \\
\hline & $\begin{array}{l}\text { No. of } \\
\text { events }\end{array}$ & $\begin{array}{l}\text { No. of } \\
\text { days }\end{array}$ & & $\begin{array}{l}\text { No. of } \\
\text { events }\end{array}$ & $\begin{array}{l}\text { No. of } \\
\text { days }\end{array}$ & $\begin{array}{l}\text { No. of } \\
\text { events }\end{array}$ & $\begin{array}{l}\text { No. of } \\
\text { days }\end{array}$ & $\begin{array}{l}\text { No. of } \\
\text { events }\end{array}$ & $\begin{array}{l}\text { No. of } \\
\text { days }\end{array}$ & $\begin{array}{l}\text { No. of } \\
\text { events }\end{array}$ & $\begin{array}{l}\text { No. of } \\
\text { days }\end{array}$ \\
\hline $\mathrm{PPCl}$ & & & No data available & & & & & N/A & & & \\
\hline $\begin{array}{l}\text { CMR centres } \\
(n=897)\end{array}$ & $6(1 \%)$ & $\begin{array}{l}14 \\
(8,17)\end{array}$ & & $112(12 \%)$ & $\begin{array}{l}79 \\
(33,205)\end{array}$ & $5(1 \%)$ & $\begin{array}{l}167 \\
(126,171)\end{array}$ & & & $2(1,3)$ & $\begin{array}{l}46 \\
(30,81)\end{array}$ \\
\hline $\begin{array}{l}\text { Non CMR centres } \\
(n=567)\end{array}$ & $4(1 \%)$ & $\begin{array}{l}20 \\
(14,34)\end{array}$ & & $33(6 \%)$ & $\begin{array}{l}45 \\
(24,116)\end{array}$ & $1(0.2 \%)$ & $\begin{array}{l}48 \\
(48,48)\end{array}$ & & & $1(0,1)$ & $\begin{array}{l}90 \\
(57,126)\end{array}$ \\
\hline \multicolumn{12}{|l|}{ MVD } \\
\hline $\begin{array}{l}\text { CMR centres } \\
(n=499)\end{array}$ & $4(1 \%)$ & $\begin{array}{l}13 \\
(8,110)\end{array}$ & & $61(12 \%)$ & $\begin{array}{l}69 \\
(32,159)\end{array}$ & $4(1 \%)$ & $\begin{array}{l}147 \\
(84,169)\end{array}$ & $53(11 \%)$ & $\begin{array}{l}71 \\
(41,151)\end{array}$ & $2(1,3)$ & $\begin{array}{l}52 \\
(32,87)\end{array}$ \\
\hline $\begin{array}{l}\text { Non CMR centres } \\
(n=227)\end{array}$ & 0 & - & & $11(5 \%)$ & $\begin{array}{l}59 \\
(15,122)\end{array}$ & 0 & - & $22(10 \%)$ & $\begin{array}{l}63 \\
(43,88)\end{array}$ & $1(0,1)$ & $\begin{array}{l}95 \\
(68,136)\end{array}$ \\
\hline LV thrombus after PPCI & & & & & & & & N/A & & & \\
\hline CMR centres $(n=1)$ & 0 & - & & 0 & - & 0 & - & & & $3(3,3)$ & $\begin{array}{l}66 \\
(66,66)\end{array}$ \\
\hline $\begin{array}{l}\text { Non CMR centres } \\
(n=0)\end{array}$ & - & - & & - & - & - & - & & & - & - \\
\hline \multicolumn{12}{|c|}{ Unobstructed coronary arteries } \\
\hline $\begin{array}{l}\text { CMR centres } \\
(n=133)\end{array}$ & $2(2 \%)$ & $\begin{array}{l}269 \\
(180,358)\end{array}$ & & $16(12 \%)$ & $\begin{array}{l}103 \\
(68,205)\end{array}$ & $4(3 \%)$ & $\begin{array}{l}162 \\
(75,254)\end{array}$ & & & $1(0,2)$ & $\begin{array}{l}65 \\
(46,103)\end{array}$ \\
\hline $\begin{array}{l}\text { Non CMR centres } \\
(n=41)\end{array}$ & 0 & - & & $2(5 \%)$ & $\begin{array}{l}184 \\
(69,299)\end{array}$ & 0 & - & & & $0(0,1)$ & $\begin{array}{l}89 \\
(54,133)\end{array}$ \\
\hline \multicolumn{12}{|l|}{$\mathrm{OHCA}$} \\
\hline $\begin{array}{l}\text { CMR centres } \\
(n=79)\end{array}$ & 0 & - & & $9(11 \%)$ & $\begin{array}{l}79 \\
(62,141)\end{array}$ & $4(5 \%)$ & $\begin{array}{l}147 \\
(99,169)\end{array}$ & & & $2(1,3)$ & $\begin{array}{l}63 \\
(42,95)\end{array}$ \\
\hline $\begin{array}{l}\text { Non CMR centres } \\
(n=30)\end{array}$ & $1(3 \%)$ & $\begin{array}{l}48 \\
(48,48)\end{array}$ & & $3(10 \%)$ & $\begin{array}{l}48 \\
(5,74)\end{array}$ & $1(3 \%)$ & $\begin{array}{l}48 \\
(48,48)\end{array}$ & & & $1(0,1)$ & $\begin{array}{l}86 \\
(52,105)\end{array}$ \\
\hline
\end{tabular}

CABG coronary artery bypass grafting, CMR cardiovascular magnetic resonance imaging, $C R T$ cardiac resynchronisation therapy, ECHO echocardiography, $H E S$ Hospital Episode Statistics, ICD implantable cardioverter defibrillator, IVUS intravascular ultrasound, IQR interquartile range, $L V$ left ventricular, $M V D$ multivessel disease, OHCA out of hospital cardiac arrest, PET positron emission tomography, PCI percutaneous coronary intervention, PEDW Patient Episode Database Wales, $\mathrm{PPCl}$ primary percutaneous coronary intervention

codes and OPCS procedure codes, identified these codes in hospital episode data for participants up to 12 months following the index procedure, and tabulated the events by patient subgroup.

We considered both the frequency of the events representing important changes in management and time to the first event for a composite primary outcome. This is because, for some changes in management (e.g. additional revascularisation for MVD patients or a new diagnosis in patients with unobstructed coronary arteries), it is the time to the event that represents the main change in management resulting from CMR. For other changes in management (e.g. additional diagnostic tests in patients with unobstructed coronaries), it is a change in the frequency/rate of an event this would represent the main change in management (e.g. a patient receiving fewer tests or outpatient appointments). We were able to tabulate the frequencies of events and their timing, and visualise these by CMR status (at the patient and centre level) for all patient subgroups. We were able to observe differences in the process of healthcare in all patient subgroups by CMR status. We did not explore possible reasons for apparent differences since this was not the aim of our study; the feasibility of implementing the consensus statements using hospital episode data was our main objective.

Composite endpoints combine several events of interest within a single outcome variable. A composite 

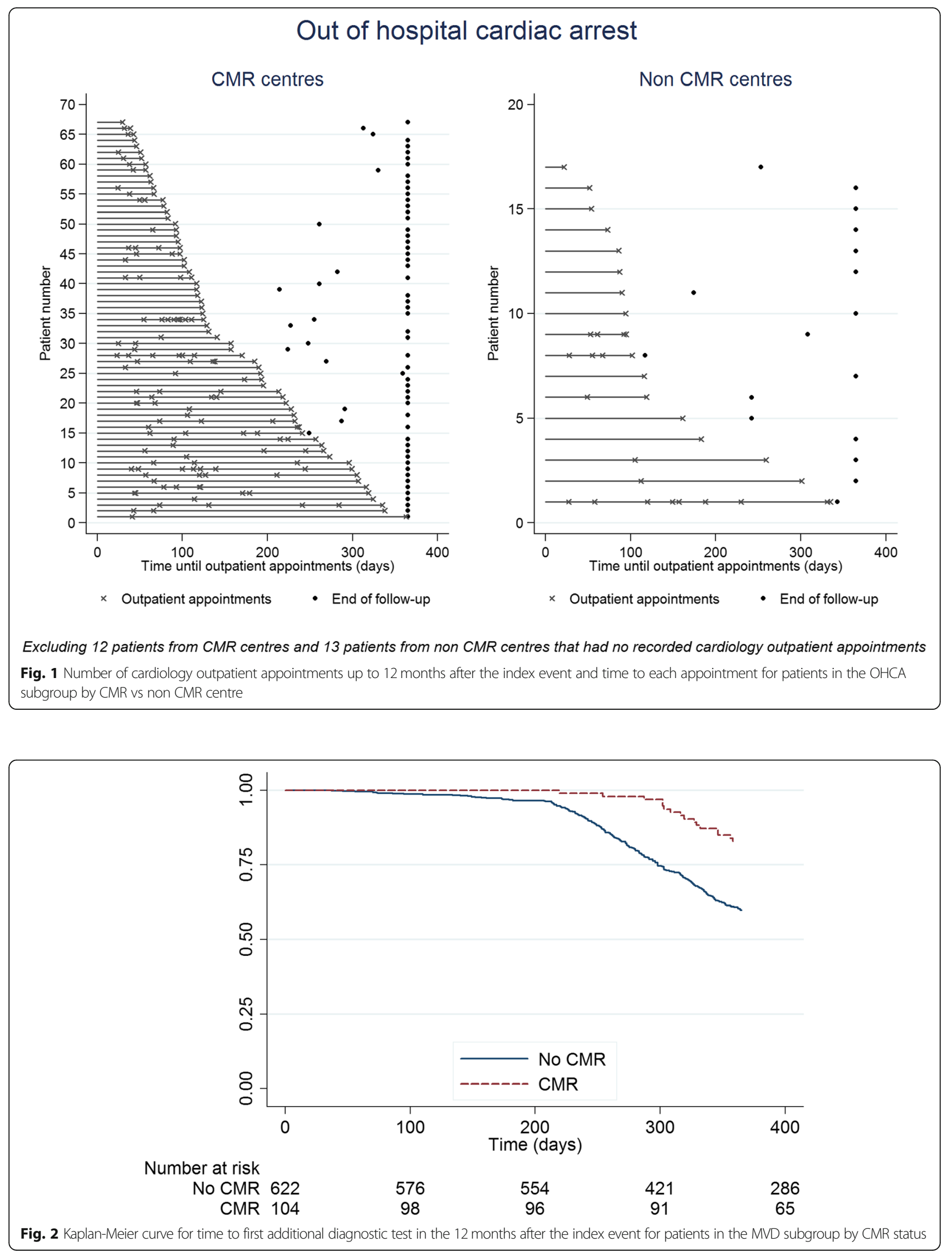
endpoint is intended to increase the frequency of a binary primary outcome and thereby increase the power of a study for a given relative treatment effect (e.g. risk, odds or hazard ratio). We did not attempt to combine clinical events to derive a composite primary outcome, either by subgroup or overall, mainly because we had no basis on which to weight different events. The derivation of a weighting algorithm to incorporate the "importance" of different clinical events would require further research, possibly involving a formal consensus process with clinicians, commissioners and patients, in order to provide a rationale for its use in a future registry assessing the clinical effectiveness of CMR in patients who activate the PPCI pathway. Another difficulty is that clinical events were characterised by different metrics (frequencies and rates) in our study, so some changes in management (e.g. the rate of cardiology outpatient appointments or additional diagnostic tests; changes in medication) cannot easily be included in a composite outcome for a time-to-event model.

\section{Strengths and limitations}

Our study has several strengths. It is based on important changes in management and hospital activities previously identified systematically using formal consensus methodology, with input from a range of all stakeholders (clinicians, methodologists and HES coders). By using routinely collected hospital data, the study benefits from an efficient design and avoids the use of primary data collection. Our decision to use HES/PEDW data rather than local hospital systems was also based on the fact that follow up data would not be available from local hospital systems for patients who are repatriated to other hospitals.

A major limitation is that we had no data on medications, either at hospital discharge or during follow up. Medications prescribed at discharge should, in principle, have been easy to obtain from participating hospitals; however, none of the hospitals had electronic pharmacy records that could be linked by a common patient identifier. Medications (either newly initiated or changes to existing prescriptions) could not be obtained during follow up because there are no medication records in any of the hospital episode datasets. There was consensus that initiation, withdrawal or changes in medication represented an important change in management for all our patient subgroups [10], in particular patients with LV thrombus for whom anticoagulation therapy is the main change in management. This limitation should be overcome in the near future with the increasing implementation and use of hospital electronic prescribing (EP) systems [15]. A potential solution to the lack of medications data during follow up is linkage of data collected from hospitals and hospital episode data with data from the Clinical Practice Research Datalink (CPRD), a primary care dataset that has detailed information on medications (https://www.cprd.com/). Only a subset of GP practices (6\%) contribute data to CPRD, so medications data would only be available for a proportion of patients in a registry.

Other changes in management (e.g. additional diagnostic tests) were likely underestimated because a large proportion of diagnostic tests are performed on an outpatient basis, and some tests, such as bedside echocardiography, are not recorded in the admitted patient care dataset. Although we could identify the frequency of outpatient appointments under the cardiology specialty (and could have done so for cardiac surgery or any other related specialty), we could not identify the tests themselves because test codes are not well recorded in outpatient datasets.

Our approach to constructing a proxy outcome reflects our original concept and appears to be feasible when the important changes in management are hypothesised to cause a change in the same direction in all events / activities included in a composite outcome for a subgroup. However, through the consensus process [10], it became apparent that some of the hypothesised changes in management arising from CMR would not necessarily result in a unidirectional change in the frequency or rate of an activity. In several instances, an important change in management was more appropriate targeting of a treatment activity to the patients who were most likely to benefit, without necessarily changing the overall frequency of the activity (potentially constrained in publicly-funded health services). For example, in patients with MVD, CMR may result in better (i.e. more cost-effective) targeting of additional revascularisation. In these situations, a simple evaluation of the frequency or rate of a proxy outcome would not detect any value of CMR to patients and the NHS. It is also worth noting that some hospital activities representing the important changes in management we identified (e.g. time to outpatient appointments and additional diagnostic tests) will be complicated by local NHS driven issues which may obscure any CMR test related differences.

Although the "concept" of creating a composite outcome reflecting changes in care management can be applied to any imaging technique for any disease condition, our composite outcome was developed from a consensus process with cardiologists which identified the changes in management resulting from CMR relevant to patients activating the PPCI pathway. The outcome cannot therefore be applied to other imaging techniques, even within the same population, since it relies on CMR being able to identify specific complications from the myocardial infarction (MI) that would not be identifiable by other imaging techniques (at least not in all relevant patient subgroups). 


\section{Conclusions}

We conclude that it is feasible to ascertain events reflecting important changes in management, their frequencies and times to first event from HES/PEDW datasets. One important changes in management (change in medication) could not be identified from HES/PEDW admission datasets or directly from hospitals; this limitation should disappear as the NHS implements more electronic systems. HES data are generally regarded as reliable and have been used to identify most of the events/activities we required [11-14]. Further work is required to validate the reliability of HES/PEDW codes for identifying patient subgroups (OHCA and LV thrombus) and the key events representing the important changes in management (for example, by comparing with local hospital data, where available) before use in a main registry. Also, further work to derive a weighting algorithm for each patient subgroup is required before attempting to combine the events in a composite endpoint. Such an endpoint would need a careful assessment of the effects on its single components and their correlations, as the observed effect of the composite does not necessarily reflect the effects of the single components and will also require different weighting algorithms for different patient subgroups.

Our composite primary outcome will not be used in a large registry (as we had anticipated when we conceived the study) because the registry itself has proved not to be feasible at present due to limitations on data availability (e.g. exposure to tests) and consent (e.g. conventional ways of obtaining consent are too time consuming for a registry with no primary data collection [9]). However, this does not invalidate the concept of creating a composite outcome that reflects changes in management as a result of having an imaging test.

\section{Additional file}

Additional file 1: ICD-10 diagnosis and OPCS procedure codes used to identify patient subgroups and changes in management in hospital episode data. (DOCX $16 \mathrm{~kb}$ )

\section{Disclaimer}

The views expressed are those of the authors and not necessarily those of the National Health Service (NHS), the National Institute for Health Research $(\mathrm{NIHR})$ or the Department of Health $(\mathrm{DoH})$.

\section{Authors' contributions}

MP developed the protocol for the study and wrote the manuscript. JH linked the datasets and conducted the statistical analysis. SD provided clinical expertise with regards to identifying the key clinical events representing the changes in management identified through formal consensus. LC provided expertise with respect to HES coding of clinical events. CBD, JPG and RA provided cardiology expertise. RB facilitated data collection for the study. BR conceived the study and provided strategic direction with regards to interpretation of study results. All authors have read and approved the final version of the manuscript.

\section{Funding}

This study is funded by the National Institute for Health Research (NIHR) Health Services and Delivery Research (HS\&DR 11/2003/58). The British Heart Foundation and NIHR Bristol Biomedical Research Centre funded some staff time (MP, JH, $\mathrm{CBD}, \mathrm{RB}, \mathrm{BCR}$ ).

\section{Availability of data and materials}

Anonymised individual patient data (excluding hospital episode/vital status linked data) can be made available for secondary research, conditional on assurance from the secondary researcher that the proposed use of the data is compliant with the MRC Policy on data preservation and sharing regarding scientific quality, ethical requirements and value for money. We are prevented from sharing hospital episode data/vital status data under our data sharing agreement with NHS Digital. Please contact maria.pufulete@bristol.ac.uk to discuss any data requests.

\section{Ethics approval and consent to participate}

Ethical approval for the cohort study was obtained from National Research Ethics Service (NRES) Committee South West-Central Bristol (reference number 12/SW/0326).

\section{Consent for publication}

Not applicable.

\section{Competing interests}

The authors declare that they have no competing interests.

\section{Author details}

${ }^{1}$ Clinical Trials and Evaluation Unit, University of Bristol, Level 7, Bristol Royal Infirmary, Queen's Building, Bristol, UK. ${ }^{2}$ NIHR Bristol Cardiovascular Research Unit, Bristol Heart Institute, University of Bristol, Bristol, UK. ${ }^{3}$ Department of Information Management \& Technology, University Hospitals Bristol NHS Foundation Trust, Bristol, UK. ${ }^{4}$ Multidisciplinary Cardiovascular Research Centre and Leeds Institute of Cardiovascular and Metabolic Medicine, University of Leeds, Leeds, UK. ${ }^{5}$ University Hospitals of Wales, Heath Park, Cardiff, UK.

Received: 8 May 2019 Accepted: 21 May 2019

Published online: 06 June 2019

References

1. Lubbeke A, Silman AJ, Barea C, Prieto-Alhambra D, Carr AJ. Mapping existing hip and knee replacement registries in Europe. Health policy. 2018; 122(5):548-57.

2. Lubbeke A, Silman AJ, Prieto-Alhambra D, Adler Al, Barea C, Carr AJ. The role of national registries in improving patient safety for hip and knee replacements. BMC Musculoskelet Disord. 2017;18(1):414.

3. Moussa I, Hermann A, Messenger JC, Dehmer GJ, Weaver WD, Rumsfeld JS, Masoudi FA. The NCDR CathPCI registry: a US national perspective on care and outcomes for percutaneous coronary intervention. Heart. 2013:99(5):297-303.

4. Prime MS, Palmer J, Khan WS. The National Joint Registry of England and Wales. Orthopedics. 2011:34(2):107-10.

5. Fox KA, Steg PG, Eagle KA, Goodman SG, Anderson FA Jr, Granger CB, Flather MD, Budaj A, Quill A, Gore JM. Decline in rates of death and heart failure in acute coronary syndromes, 1999-2006. Jama. 2007;297(17):1892-900. 
6. Puymirat E, Simon T, Steg PG, Schiele F, Gueret P, Blanchard D, Khalife K, Goldstein P, Cattan S, Vaur L, et al. Association of changes in clinical characteristics and management with improvement in survival among patients with ST-elevation myocardial infarction. Jama. 2012;308(10):998-1006.

7. Rogers WJ, Frederick PD, Stoehr E, Canto JG, Ornato JP, Gibson CM, Pollack CV Jr, Gore JM, Chandra-Strobos N, Peterson ED, et al. Trends in presenting characteristics and hospital mortality among patients with ST elevation and non-ST elevation myocardial infarction in the National Registry of myocardial infarction from 1990 to 2006. Am Heart J. 2008;156(6):1026-34.

8. Yeh RW, Sidney S, Chandra M, Sorel M, Selby JV, Go AS. Population trends in the incidence and outcomes of acute myocardial infarction. N Engl J Med. 2010;362(23):2155-65.

9. Brierley RC, Pufulete M, Harris J, Bucciarelli-Ducci C, Greenwood JP, Dorman $\mathrm{S}$, Anderson R, Rogers CA, Reeves BC. Developing a UK registry to investigate the role of cardiovascular magnetic resonance (CMR) in patients who activate the primary percutaneous coronary intervention (PPCI) pathway: a multicentre, feasibility study linking routinely collected electronic patient data. BMJ Open. 2018;8(3):e018987.

10. Pufulete M, Brierley RC, Bucciarelli-Ducci C, Greenwood JP, Dorman S, Anderson RA, Harris J, McAlindon E, Rogers CA, Reeves BC. Formal consensus to identify clinically important changes in management resulting from the use of cardiovascular magnetic resonance (CMR) in patients who activate the primary percutaneous coronary intervention (PPCI) pathway. BMJ Open. 2017:7(6):e014627.

11. Hospital episode statistics and revalidation: creating the evidence to support revalidation. Royal College of Surgeons of England, 2013.

12. Bortolussi G, McNulty D, Waheed H, Mawhinney JA, Freemantle N, Pagano D. Identifying cardiac surgery operations in hospital episode statistics administrative database, with an OPCS-based classification of procedures, validated against clinical data. BMJ Open. 2019;9(3):e023316.

13. Kivimaki M, Batty GD, Singh-Manoux A, Britton A, Brunner EJ, Shipley MJ Validity of cardiovascular disease event ascertainment using linkage to UK Hospital records. Epidemiology. 2017;28(5):735-9.

14. Barry SJ, Dinnett E, Kean S, Gaw A, Ford I. Are routinely collected NHS administrative records suitable for endpoint identification in clinical trials? Evidence from the west of Scotland coronary prevention study. PLoS One. 2013;8(9):e75379.

15. HM Government. Personalised health and care 2020. 2014. www.gov.uk government/uploads/system/uploads/attachment_data/file/384650/NIB_ Report.pdf.

\section{Publisher's Note}

Springer Nature remains neutral with regard to jurisdictional claims in published maps and institutional affiliations.

Ready to submit your research? Choose BMC and benefit from:

- fast, convenient online submission

- thorough peer review by experienced researchers in your field

- rapid publication on acceptance

- support for research data, including large and complex data types

- gold Open Access which fosters wider collaboration and increased citations

- maximum visibility for your research: over $100 \mathrm{M}$ website views per year

At $\mathrm{BMC}$, research is always in progress.

Learn more biomedcentral.com/submissions 